\title{
Unique Solution of a Coupled Fractional Differential System Involving Integral Boundary Conditions from Economic Model
}

\author{
Rui Li, ${ }^{1}$ Haoqian Zhang, ${ }^{2}$ and Hao Tao ${ }^{3,4}$ \\ ${ }^{1}$ Department of Electrical Engineering, North China Electric Power University, Baoding 071003, China \\ ${ }^{2}$ Department of Management and Economic, North China Electric Power University, Baoding 071003, China \\ ${ }^{3}$ School of Land Science and Technology, China University of Geosciences, Beijing 100083, China \\ ${ }^{4}$ Institute of Finance and Banking, Chinese Academy of Social Sciences, Beijing, China \\ Correspondence should be addressed to Rui Li; dianlilirui@sina.com and Hao Tao; taohao512@gmail.com
}

Received 13 May 2013; Accepted 1 July 2013

Academic Editor: Yonghong Wu

Copyright (C) 2013 Rui Li et al. This is an open access article distributed under the Creative Commons Attribution License, which permits unrestricted use, distribution, and reproduction in any medium, provided the original work is properly cited.

We study the existence and uniqueness of the positive solution for the fractional differential system involving the Riemann-Stieltjes integral boundary conditions $-\mathscr{D}_{t}^{\alpha} x(t)=f(t, y(t)),-\mathscr{D}_{t}^{\beta} y(t)=g(t, x(t)), t \in(0,1), x(0)=y(0)=0, x(1)=\int_{0}^{1} x(s) d A(s)$, and $y(1)=\int_{0}^{1} y(s) d B(s)$, where $1<\alpha, \beta \leq 2$, and $\mathscr{D}_{t}^{\alpha}$ and $\mathscr{D}_{t}^{\beta}$ are the standard Riemann-Liouville derivatives, $A$ and $B$ are functions of bounded variation, and $\int_{0}^{1} \mathscr{D}_{t}^{\beta} x(s) d A(s)$ and $\int_{0}^{1} \mathscr{D}_{t}^{\beta} y(s) d B(s)$ denote the Riemann-Stieltjes integral. Our results are based on a generalized fixed point theorem for weakly contractive mappings in partially ordered sets.

\section{Introduction}

Mathematical model is an important tool designed to describe the operation of the economy of a country or a region. Because fractional operators are non-local, they are more suitable for constructing models possessing memory effect with the long time periods, and then fractional differential equations possess large advantage in describing economic phenomena over the time periods. In this paper, we focus on a fractional model arising from economy. Assuming that $f, g:[0,1] \times[0,+\infty) \rightarrow[0,+\infty)$ are continuous and nondecreasing with respect to the second variable on $[0,+\infty)$, we discuss the existence and uniqueness of positive solutions for the following system of fractional differential equation with nonlocal Riemann-Stieltjes integral boundary conditions:

$$
\begin{gathered}
-\mathscr{D}_{t}^{\alpha} x(t)=f(t, y(t)), \\
-\mathscr{D}_{t}^{\beta} y(t)=g(t, x(t)), \quad t \in(0,1),
\end{gathered}
$$

$$
\begin{gathered}
x(0)=y(0)=0, \quad x(1)=\int_{0}^{1} x(s) d A(s), \\
y(1)=\int_{0}^{1} y(s) d B(s),
\end{gathered}
$$

where $1<\alpha, \beta \leq 2$, and $\mathscr{D}_{t}^{\alpha}$ and $\mathscr{D}_{t}^{\beta}$ are the standard Riemann-Liouville derivatives, $A$ and $B$ are functions of bounded variation, and $\int_{0}^{1} \mathscr{D}_{t}^{\beta} x(s) d A(s)$ and $\int_{0}^{1} \mathscr{D}_{t}^{\beta} y(s) d B(s)$ denote the Riemann-Stieltjes integral.

Boundary value problems with integral boundary conditions constitute a very interesting and important class of problems. The idea of using a Riemann-Stieltjes integral with a signed measure is due to Webb and Infante in [1, $2]$. The papers [1-3] contain several new ideas and give a unified approach to many BVPs. This implies that the Riemann-Stieltjes integral boundary value problem is a more generalized case which includes multipoints, integral boundary conditions and many nonlocal boundary conditions, as special cases. For some recent work on boundary value 
problems of fractional differential equation, we refer the reader to some recent papers (see [1-14]).

Recently, motivated by [1-3], Hao et al. [4] studied the existence of positive solutions for the $n$ th-order singular nonlocal boundary value problem

$$
\begin{gathered}
x^{(n)}(t)+a(t) f(t, x(t))=0, \quad 0<t<1, \\
x^{(k)}(0)=0, \quad 0 \leq k \leq n-2, \quad x(1)=\int_{0}^{1} x(s) d A(s),
\end{gathered}
$$

where $a$ may be singular at $t=0,1$ and $f$ also may be singular at $x=0$, but there is no singularity at $t=0,1$. The existence of positive solutions of the BVP (2) is obtained by means of the fixed point index theory in cones. In [5], Zhang and Han considered the existence of positive solutions of the following singular fractional differential equation:

$$
\begin{aligned}
& \mathscr{D}_{t}^{\alpha} x(t)+f(t, x(t))=0, \quad 0<t<1, n-1<\alpha \leq n, \\
& x^{(k)}(0)=0, \quad 0 \leq k \leq n-2, \quad x(1)=\int_{0}^{1} x(s) d A(s),
\end{aligned}
$$

where $\alpha \geq 2$, and $d A(s)$ can be a signed measure. Some growth conditions were adopted to guarantee that (3) has a unique positive solution. Recently, by using a new fixed point theorem for weakly contractive mappings in partially ordered sets, Tao et al. [6] considered the existence and uniqueness of positive solution for the following problem:

$$
\begin{gathered}
-\mathscr{D}_{t}^{\alpha} x(t)=f\left(t, x(t),-\mathscr{D}_{t}^{\beta} x(t)\right), \quad t \in(0,1), \\
\mathscr{D}_{t}^{\beta} x(0)=\mathscr{D}_{t}^{\beta+1} x(0)=0, \\
\mathscr{D}_{t}^{\beta} x(1)=\int_{0}^{1} \mathscr{D}_{t}^{\beta} x(s) d A(s),
\end{gathered}
$$

where $2<\alpha \leq 3,0<\beta<1$, and $\alpha-\beta>2$, and $\mathscr{D}_{t}$ is the standard Riemann-Liouville derivative.

Motivated by the above work, in this paper, we study the existence and uniqueness of positive solution for the system of fractional differential equations with nonlocal RiemannStieltjes boundary integral conditions. Our main tool is the fixed point theorem for weakly contractive mappings in partially ordered sets, which is obtained by papers $[6,15]$.

\section{Preliminaries and Lemmas}

Definition 1 (see [16-18]). The Riemann-Liouville fractional integral of order $\alpha>0$ of a function $x:(0,+\infty) \rightarrow \mathbb{R}$ is given by

$$
I^{\alpha} x(t)=\frac{1}{\Gamma(\alpha)} \int_{0}^{t}(t-s)^{\alpha-1} x(s) d s
$$

provided that the right-hand side is pointwise defined on $(0,+\infty)$.
Definition 2 (see [16-18]). The Riemann-Liouville fractional derivative of order $\alpha>0$ of a function $x:(0,+\infty) \rightarrow \mathbb{R}$ is given by

$$
\mathscr{D}_{t}^{\alpha} x(t)=\frac{1}{\Gamma(n-\alpha)}\left(\frac{d}{d t}\right)^{n} \int_{0}^{t}(t-s)^{n-\alpha-1} x(s) d s,
$$

where $n=[\alpha]+1,[\alpha]$ denotes the integer part of number $\alpha$, provided that the right-hand side is pointwise defined on $(0,+\infty)$.

Lemma 3 (see [16-18]). (1) If $x \in L^{1}(0,1), v>\sigma>0$, then

$$
\begin{gathered}
I^{\nu} I^{\sigma} x(t)=I^{\nu+\sigma} x(t), \quad \mathscr{D}_{t}^{\sigma} I^{\nu} x(t)=I^{\nu-\sigma} x(t), \\
\mathscr{D}_{t}^{\sigma} I^{\sigma} x(t)=x(t) .
\end{gathered}
$$

(2) If $v>0, \sigma>0$, then

$$
\mathscr{D}_{t}^{v} t^{\sigma-1}=\frac{\Gamma(\sigma)}{\Gamma(\sigma-\nu)} t^{\sigma-\nu-1} .
$$

(3) If $\alpha>0$ and $f(x)$ is integrable, then

$$
I^{\alpha} \mathscr{D}_{t}^{\alpha} x(t)=f(x)+c_{1} x^{\alpha-1}+c_{2} x^{\alpha-2}+\cdots+c_{n} x^{\alpha-n},
$$

where $c_{i} \in \mathbb{R}(i=1,2, \ldots, n), n$ is the smallest integer greater than or equal to $\alpha$.

Lemma 4 (see [19]). Given $h \in L^{1}(0,1)$. Then, the problems

$$
\begin{aligned}
& \mathscr{D}_{t}^{\alpha} x(t)+h(t)=0, \quad t \in(0,1), \quad x(0)=0, \quad x(1)=0, \\
& \mathscr{D}_{t}^{\beta} y(t)+h(t)=0, \quad t \in(0,1), \quad y(0)=0, \quad y(1)=0
\end{aligned}
$$

have the unique solution

$$
x(t)=\int_{0}^{1} G_{A}(t, s) h(s) d s, \quad y(t)=\int_{0}^{1} G_{B}(t, s) h(s) d s,
$$

where $G_{A}(t, s)$ and $G_{B}(t, s)$ are given by

$$
\begin{aligned}
& G_{A}(t, s)=\frac{1}{\Gamma(\alpha)} \begin{cases}{[t(1-s)]^{\alpha-1},} & t \leq s, \\
{[t(1-s)]^{\alpha-1}-(t-s)^{\alpha-1},} & s \leq t,\end{cases} \\
& G_{B}(t, s)=\frac{1}{\Gamma(\beta)} \begin{cases}{[t(1-s)]^{\beta-1},} & t \leq s, \\
{[t(1-s)]^{\beta-1}-(t-s)^{\beta-1},} & s \leq t,\end{cases}
\end{aligned}
$$

which are the Green function of the BVP (10), respectively.

It follows from Lemma 3 that the linear problems

$$
\begin{aligned}
& \mathscr{D}_{t}^{\alpha} x(t)=0, \quad 0<t<1, \quad x(0)=0, \quad x(1)=1, \\
& \mathscr{D}_{t}^{\beta} y(t)=0, \quad 0<t<1, \quad y(0)=0, \quad y(1)=1
\end{aligned}
$$

have the unique solutions $t^{\alpha-1}$ and $t^{\beta-1}$, respectively. Letting

$$
\mathscr{A}=\int_{0}^{1} t^{\alpha-1} d A(t), \quad \mathscr{B}=\int_{0}^{1} t^{\beta-1} d B(t)
$$


and defining

$$
\mathscr{G}_{A}(s)=\int_{0}^{1} G(t, s) d A(t), \quad \mathscr{G}_{B}(s)=\int_{0}^{1} G(t, s) d B(t)
$$

as in [3-5], we can get that the Green functions for nonlocal BVP (1) are given by

$$
\begin{aligned}
& H_{A}(t, s)=\frac{t^{\alpha-1}}{1-\mathscr{A}} \mathscr{G}_{A}(s)+G_{A}(t, s), \\
& H_{B}(t, s)=\frac{t^{\beta-1}}{1-\mathscr{B}} \mathscr{G}_{B}(s)+G_{B}(t, s) .
\end{aligned}
$$

To ensure the nonnegativity of Green functions, we use the following elementary assumption in this paper.

(H0) $A, B$ are increasing functions of bounded variation such that $\mathscr{G}_{A}(s) \geq 0, \mathscr{G}_{B}(s) \geq 0$ for $s \in[0,1]$ and $0 \leq \mathscr{A}, \mathscr{B}<1$, where $\mathscr{A}, \mathscr{B}$ are defined by (14).

Lemma 5. Let $1<\alpha, \beta \leq 2$, and let $(H 0)$ hold; then $H_{A}(t, s)$ and $H_{B}(t, s)$ satisfy

$$
\begin{aligned}
& 0 \leq H_{A}(t, s) \leq \frac{1}{(1-\mathscr{A}) \Gamma(\alpha-1)}=\mathscr{A}^{*}, \\
& 0 \leq H_{B}(t, s) \leq \frac{1}{(1-\mathscr{B}) \Gamma(\beta-1)}=\mathscr{B}^{*} .
\end{aligned}
$$

The main tool of this paper is the following fixed point theorem, which was firstly obtained by Harjani and Sadarangani [15] and was improved by Tao et al. [6].

Lemma 6 (see $[6])$. Let $(X, \geq)$ be a partially ordered set, and suppose that there exists a metric $d$ in $X$ such that $(X, d)$ is a complete metric space. Assume that $X$ satisfies the following condition: if $x_{n}$ is a nondecreasing sequence in $X$ such that $x_{n} \rightarrow x$, then $x_{n} \leq x$ for all $n \in \mathbb{N}$. Let $T: X \rightarrow X$ be a nondecreasing mapping, and there exists a constant $\lambda \in(0,1)$ such that

$$
d(T x, T y) \leq \lambda d(x, y)-\psi(\lambda d(x, y)), \quad \text { for } x \geq y,
$$

where $\psi:[0,+\infty) \rightarrow[0,+\infty)$ is a nondecreasing function. If there exists $x \in X$ with $x_{0} \leq T x_{0}$, then $T$ has a fixed point.

If the space $(X, \leq)$ satisfies the following condition:

for $x, y \in X$, there exists $z \in X$

which is comparable to $x$ and $y$,

then we have the following theorem; see $[6,15]$.

Lemma 7. Adding condition (19) to the hypotheses of Lemma 6, one obtains uniqueness of the fixed point of T.

Now, we mean by $C[0,1]$ the Banach space of all continuous functions on $[0,1]$ with the usual $\|x\|=\max _{0 \leq t \leq 1}|x(t)|$.
Note that this space can be equipped with a partial order given by

$$
\begin{array}{r}
x, y \in C[0,1], \quad x \leq y \Longleftrightarrow x(t) \leq y(t), \\
\text { for } t \in[0,1] .
\end{array}
$$

It has been proved in $[20,21]$ that $(C[0,1], \leq)$ with the classic metric given by

$$
d(x, y)=\max _{0 \leq t \leq 1}\{|x(t)-y(t)|\}
$$

satisfies the following condition.

If $x_{n}$ is a nondecreasing sequence in $X$ such that $x_{n} \rightarrow$ $x$, then $x_{n} \leq x$ for all $n \in \mathbb{N}$. Moreover, for $x, y \in C[0,1]$, the function $\max \{x, y\}$ is continuous in $[0,1]$, and $(C[0,1], \leq)$ satisfies condition (19).

Next, define a subcone of $C[0,1]$ as follows:

$$
P=\{x \in C[0,1]: x(t) \geq 0\} .
$$

Note that $P$ is a closed set of $C[0,1] ; P$ is a complete metric space.

Clearly, $(x, y)$ is a solution of system (1) if and only if $(x, y) \in C[0,1] \times C[0,1]$ is a solution of the following nonlinear integral system of equations:

$$
\begin{aligned}
& x(t)=\int_{0}^{1} H_{A}(t, s) f(s, y(s)) d s, \\
& y(t)=\int_{0}^{1} H_{B}(t, s) g(s, x(s)) d s .
\end{aligned}
$$

Consequently, system (23) is equivalent to the following integral equation:

$$
x(t)=\int_{0}^{1} H_{A}(t, s) f\left(s, \int_{0}^{1} H_{B}(s, \tau) g(\tau, x(\tau)) d \tau\right) d s .
$$

Thus, for $x \in P$, define the operator $T$ by

$$
(T x)(t)=\int_{0}^{1} H_{A}(t, s) f\left(s, \int_{0}^{1} H_{B}(s, \tau) g(\tau, x(\tau)) d \tau\right) d s .
$$

Then from the assumption on $f, g$, and Lemma 5, we have $T(P) \subset P$.

\section{Main Results}

Now, define the class of functions $\mathscr{X}$,

$$
\begin{aligned}
& \mathscr{X}=\{\phi \mid \phi:[0,+\infty) \longrightarrow[0,+\infty) \\
& \quad \text { is differential and nondecreasing, } \\
& \text { and } \psi(x)=x-\phi(x) \text { satisfies } \\
& \\
& \psi:[0,+\infty) \longrightarrow[0,+\infty) \text { is nondecreasing }\} .
\end{aligned}
$$


Remark 8. The standard function $\phi \in \mathscr{X}$; for example, $\phi(x)=$ $\arctan x, \phi(x)=(1 / 2) x, \phi(x)=\ln (1+x), \phi(x)=x /(1+x)$, and so forth.

Remark 9. Clearly, given that $\phi_{1}, \phi_{2} \in \mathscr{X}$, then $\phi_{1}\left(\phi_{2}(x)\right) \in$ $x$.

Theorem 10. Suppose that (H0) holds, and there exist two functions $\phi_{1}, \phi_{2} \in \mathscr{X}$ and constants $\rho_{1}, \rho_{2}, \theta_{1}$, and $\theta_{2}$ which satisfy

$$
\begin{aligned}
& 0<\rho_{1} \leq \mathscr{A}^{*-1}, \quad 0<\rho_{2} \leq \mathscr{B}^{*-1}, \\
& 0<\theta_{1}<\rho_{2}^{-1} \mathscr{B}^{*-1}, \quad 0<\theta_{2}<1
\end{aligned}
$$

such that

$$
\begin{aligned}
& f\left(t, x_{1}\right)-f\left(t, x_{2}\right) \leq \rho_{1} \phi_{1}\left(\theta_{1}\left(x_{1}-x_{2}\right)\right), \\
& g\left(t, y_{1}\right)-g\left(t, y_{2}\right) \leq \rho_{2} \phi_{2}\left(\theta_{2}\left(y_{1}-y_{2}\right)\right),
\end{aligned}
$$

for $x_{1}, x_{2}, y_{1}, y_{2} \in[0,+\infty)$ with $x_{1} \geq x_{2}, y_{1} \geq y_{2}$. Then, problem (1) has a unique nonnegative solution.

Proof. To prove that the problem (1) has a unique nonnegative solution, it is sufficient to check that the hypotheses of Lemma 6 are satisfied.

By the monotonicity of $f$ and $g$, we know that the operator $T$ is nondecreasing. Now, denote

$$
\phi(x)=\phi_{1}\left(\phi_{2}(x)\right) .
$$

Then, $\phi \in \mathscr{X}$. For any $u \geq v \in P$, from (27) and (28), we have

$$
\begin{aligned}
& d(T u, T v) \\
& =\max _{t \in[0,1]}|T u(t)-T v(t)| \\
& =\max _{t \in[0,1]} \int_{0}^{1} H_{A}(t, s) \\
& \times\left[f\left(s, \int_{0}^{1} H_{B}(s, \tau) g(\tau, u(\tau)) d \tau\right)\right. \\
& \left.\quad-f\left(s, \int_{0}^{1} H_{B}(s, \tau) g(\tau, v(\tau)) d \tau\right)\right] d s \\
& \leq \max _{t \in[0,1]}\left\{\int_{0}^{1} H_{A}(t, s)\right. \\
& \quad \times\left[\rho _ { 1 } \phi _ { 1 } \left(\theta_{1} \int_{0}^{1} H_{B}(s, \tau)\right.\right. \\
& \leq \mathscr{A}^{*} \rho_{1} \phi_{1}\left(\mathscr{B}^{*} \theta_{1} \rho_{2} \phi_{2}\left(\theta_{2}(u(\tau))-v(\tau)\right)\right) \\
& \leq \mathscr{A}^{*} \rho_{1} \phi_{1}\left(\phi_{2}\left(\theta_{2} d(u, v)\right) \leq \phi\left(\theta_{2} d(u, v)\right) .\right. \\
& -g(\tau, v(\tau))))] d s\}
\end{aligned}
$$

Since $\phi \in \mathscr{X}$, which implies that $\psi(x)=x-\phi(x)$, and $\psi$ : $[0,+\infty) \rightarrow[0,+\infty)$ is nondecreasing. Thus, for $u \geq v$, we find $\psi$ and $\theta_{2}$ such that

$$
\begin{aligned}
d(T u, T v) & \leq \phi\left(\theta_{2} d(u, v)\right) \\
& =\theta_{2} d(u, v)-\left(\theta_{2} d(u, v)-\phi\left(\theta_{2} d(u, v)\right)\right) \\
& =\theta_{2} d(u, v)-\psi\left(\theta_{2} d(u, v)\right) .
\end{aligned}
$$

Finally, taking into account the zero function, $0 \leq$ $(T 0)(t)$, by Lemma 6, problem (1) has a unique nonnegative solution.

In the following, we consider the positive solution of the problem (1). A positive solution $(x, y)$ of the problem (1) means a solution of the problem (1) satisfying $x(t)>0$, $y(t)>0$ for $t \in(0,1)$.

Theorem 11. Suppose that there exists $\hat{t} \in[0,1]$ such that $f(\widehat{t}, 0) \neq 0$ and $g(\hat{t}, 0) \neq 0$, and the assumptions of Theorem 10 also hold; then, the unique solution of (1) is positive.

Proof. It follows from Theorem 10 that the problem (1) has a unique nonnegative solution. We prove that it is also a positive solution of the problem (1).

Otherwise, there exists $0<t^{*}<1$ such that $x\left(t^{*}\right)=0$; that is;

$$
\begin{aligned}
x\left(t^{*}\right)= & \int_{0}^{1} H_{A}\left(t^{*}, s\right) f \\
& \times\left(s, \int_{0}^{1} H_{B}(s, \tau) g(\tau, x(\tau)) d \tau\right) d s=0 .
\end{aligned}
$$

Then,

$$
\begin{aligned}
0=x\left(t^{*}\right)= & \int_{0}^{1} H_{A}\left(t^{*}, s\right) f \\
& \times\left(s, \int_{0}^{1} H_{B}(s, \tau) g(\tau, x(\tau)) d \tau\right) d s \\
\geq & \int_{0}^{1} H_{A}\left(t^{*}, s\right) f(s, 0) d s \geq 0 .
\end{aligned}
$$

Consequently,

$$
\int_{0}^{1} H_{A}\left(t^{*}, s\right) f(s, 0) d s=0 .
$$

Note that $H\left(t^{*}, s\right)>0, s \in(0,1)$; then, we have

$$
f(s, 0)=0, \quad \text { a.e. }(0,1) \text {. }
$$

In addition, it follows from $f(\hat{t}, 0) \neq 0, \widehat{t} \in[0,1]$ that $f(\widehat{t}, 0)>0$, and by the continuity of $f$, we can find a set $\Omega \subset[0,1]$ such that $\hat{t} \in \Omega$ and $f(t, 0)>0$ for any $t \in \Omega$, where $\mu(\Omega)>0$ and $\mu(\cdot)$ stands for the Lebesgue measure, which contradicts with (35). Consequently, we have $x(t)>0$, $t \in(0,1)$. In the same way, we also have

$$
y(t)=\int_{0}^{1} H_{B}(t, s) g(s, x(s)) d s
$$


and $y(t)>0, t \in(0,1)$. Hence, the problem (1) has a unique positive solution.

Example 12. Consider the following boundary value problem with fractional order $\alpha=1.5, \beta=1.2$ :

$$
\begin{gathered}
-\mathscr{D}_{t}^{1.5} x(t)=t^{2} \sin t+\frac{y(t)}{100(1+y(t))}, \\
-\mathscr{D}_{t}^{1.2} y(t)=t e^{t}+\frac{1}{300} x(t), \quad 0<t<1, \\
x(0)=y(0)=0, \quad x(1)=\int_{0}^{1} x(s) d A(s), \\
y(1)=\int_{0}^{1} y(s) d B(s),
\end{gathered}
$$

where

$$
\begin{aligned}
& A(t)= \begin{cases}0, & t \in\left[0, \frac{1}{3}\right), \\
\frac{1}{6}, & t \in\left[\frac{1}{3}, \frac{2}{3}\right), \\
\frac{1}{2}, & t \in\left[\frac{2}{3}, 1\right],\end{cases} \\
& B(t)= \begin{cases}0, & t \in\left[0, \frac{1}{4}\right), \\
\frac{1}{2}, & t \in\left[\frac{1}{4}, \frac{3}{4}\right), \\
\frac{1}{3}, & t \in\left[\frac{3}{4}, 1\right] .\end{cases}
\end{aligned}
$$

The BVP (37) becomes the 4-point BVP with coefficients:

$$
\begin{gathered}
-\mathscr{D}_{t}^{1.5} x(t)=t^{2} \sin t+\frac{y(t)}{100(1+y(t))}, \\
-\mathscr{D}_{t}^{1.2} y(t)=t e^{t}+\frac{1}{300} x(t), \quad 0<t<1, \\
x(0)=y(0)=0, \quad x(1)=\frac{1}{6} x\left(\frac{2}{3}\right)+\frac{1}{3} x\left(\frac{1}{3}\right), \\
y(1)=\frac{1}{2} y\left(\frac{1}{4}\right)+\frac{1}{6} y\left(\frac{3}{4}\right) .
\end{gathered}
$$

By Theorem 11, the BVP (37) has a unique positive solution.

Proof. Obviously, $\alpha=1.5, \beta=1.2$, and

$$
\begin{aligned}
& \mathscr{A}=\int_{0}^{1} t^{0.5} d A(t)=\frac{1}{6}\left(\frac{1}{3}\right)^{0.5}+\frac{1}{3}\left(\frac{2}{3}\right)^{0.5} \approx 0.3684<1, \\
& \mathscr{B}=\int_{0}^{1} t^{0.2} d B(t)=\frac{1}{2}\left(\frac{1}{4}\right)^{0.2}+\frac{1}{6}\left(\frac{3}{4}\right)^{0.2} \approx 0.8171<1, \\
& \mathscr{A}^{*-1}=(1-\mathscr{A}) \Gamma(0.5)=1.1195, \\
& \mathscr{B}^{*-1}=(1-\mathscr{B}) \Gamma(0.2)=0.8397 .
\end{aligned}
$$

By simple calculation, we know that $\mathscr{G}_{A}(s) \geq 0, \mathscr{G}_{B}(s) \geq 0$, and $A(s), B(s)$ are all increasing. So, $(\mathrm{H} 0)$ holds.

Take

$$
\begin{gathered}
f(t, u)=t^{2} \sin t+\frac{u}{100(1+u)}, \\
g(t, u)=t e^{t}+\frac{1}{300} u, \\
(t, u) \in[0,1] \times[0,+\infty), \\
\phi_{1}(x)=\frac{x}{1+x}, \quad \phi_{2}(x)=\frac{1}{2} x .
\end{gathered}
$$

Then, for any $x_{1} \geq x_{2}, y_{1} \geq y_{2}$,

$$
\begin{aligned}
f\left(t, x_{1}\right)-f\left(t, x_{2}\right)= & \frac{x_{1}}{100\left(1+x_{1}\right)}-\frac{x_{2}}{100\left(1-x_{2}\right)} \\
\leq & \frac{x_{1}-x_{2}}{100\left(1+x_{1}-x_{2}\right)} \\
\leq & \frac{1}{100} \times \frac{x_{1}-x_{2}}{1+(1 / 2)\left(x_{1}-x_{2}\right)}+\frac{1}{300} \\
& \times \frac{y_{2}-y_{1}}{1+(1 / 3)\left(y_{2}-y_{1}\right)} \\
= & \frac{1}{50} \times \frac{(1 / 2)\left(x_{1}-x_{2}\right)}{1+(1 / 2)\left(x_{1}-x_{2}\right)} \\
= & \frac{1}{50} \phi_{1}\left(\frac{1}{2}\left(x_{1}-x_{2}\right)\right), \\
g\left(t, y_{1}\right)-g\left(t, y_{2}\right)= & \frac{1}{300}\left(y_{1}-y_{2}\right) \\
\leq & \frac{1}{2}\left[\frac{1}{50}\left(\frac{1}{2}\left(y_{1}-y_{1}\right)\right)\right],
\end{aligned}
$$

where

$$
\begin{gathered}
\rho_{1}=\frac{1}{50}<1.1195=\mathscr{A}^{*-1}, \quad \rho_{2}=\frac{1}{2}<0.8397=\mathscr{B}^{*-1}, \\
\theta_{1}=\frac{1}{2}<1.6794=\rho_{2}^{-1} \mathscr{B}^{*-1}, \quad \theta_{2}=\frac{1}{50}<1 .
\end{gathered}
$$

Thus, $\phi_{1}, \phi_{2} \in \mathscr{X}$, and all of the conditions of Theorem 10 are satisfied.

On the other hand, $f(\pi / 6,0)=\pi^{2} / 36 \neq 0, g(1 / 2,0)=$ $(1 / 2) e^{1 / 2} \neq 0$, and by Theorem 11 , the BVP (37) has a unique positive solution.

\section{References}

[1] J. R. L. Webb and G. Infante, "Positive solutions of nonlocal boundary value problems involving integral conditions," Nonlinear Differential Equations and Applications, vol. 15, no. 1-2, pp. 45-67, 2008.

[2] J. R. L. Webb and G. Infante, "Positive solutions of nonlocal boundary value problems: a unified approach," Journal of the London Mathematical Society, vol. 74, no. 3, pp. 673-693, 2006. 
[3] J. R. L. Webb, "Nonlocal conjugate type boundary value problems of higher order," Nonlinear Analysis. Theory, Methods \& Applications A, vol. 71, no. 5-6, pp. 1933-1940, 2009.

[4] X. Hao, L. Liu, Y. Wu, and Q. Sun, "Positive solutions for nonlinear $n$ th-order singular eigenvalue problem with nonlocal conditions," Nonlinear Analysis. Theory, Methods \& Applications A, vol. 73, no. 6, pp. 1653-1662, 2010.

[5] X. Zhang and Y. Han, "Existence and uniqueness of positive solutions for higher order nonlocal fractional differential equations," Applied Mathematics Letters, vol. 25, no. 3, pp. 555-560, 2012.

[6] H. Tao, M. Fu, and R. Qian, "Positive solutions for fractional differential equations from real estate asset securitization via new fixed point theorem," Abstract and Applied Analysis, vol. 2012, Article ID 842358, 11 pages, 2012.

[7] X. Zhang, L. Liu, and Y. Wu, "The uniqueness of positive solution for a singular fractional differential system involving derivatives," Communications in Nonlinear Science and Numerical Simulation, vol. 18, pp. 1400-1409, 2013.

[8] X. Zhang, L. Liu, and Y. Wu, "Multiple positive solutions of a singular fractional differential equation with negatively perturbed term," Mathematical and Computer Modelling, vol. 55, no. 3-4, pp. 1263-1274, 2012.

[9] X. Zhang, L. Liu, and Y. Wu, "The eigenvalue problem for a singular higher order fractional differential equation involving fractional derivatives," Applied Mathematics and Computation, vol. 218, no. 17, pp. 8526-8536, 2012.

[10] W. Wang and L. Huang, "Existence of positive solution for semipositone fractional differential equations involving RiemannStieltjes integral conditions," Abstract and Applied Analysis, vol. 2012, Article ID 723507, 17 pages, 2012.

[11] X. Zhang, L. Liu, and Y. Wu, "Existence results for multiple positive solutions of nonlinear higher order perturbed fractional differential equations with derivatives," Applied Mathematics and Computation, vol. 219, no. 4, pp. 1420-1433, 2012.

[12] X. Zhang, L. Liu, Y. Wu, and Y. Lu, "The iterative solutions of nonlinear fractional differential equations," Applied Mathematics and Computation, vol. 219, no. 9, pp. 4680-4691, 2013.

[13] X. Zhang, L. Liu, B. Wiwatanapataphee, and Y. Wu, "Positive solutions of eigenvalue problems for a class of fractional differential equations with derivatives," Abstract and Applied Analysis, vol. 2012, Article ID 512127, 16 pages, 2012.

[14] M. Jia, X. Zhang, and X. Gu, "Nontrivial solutions for a higher fractional differential equation with fractional multipoint boundary conditions," Boundary Value Problems, vol. 2012, article 70, 2012.

[15] J. Harjani and K. Sadarangani, "Fixed point theorems for weakly contractive mappings in partially ordered sets," Nonlinear Analysis. Theory, Methods \& Applications A, vol. 71, no. 7-8, pp. 34033410, 2009.

[16] K. S. Miller and B. Ross, An Introduction to the Fractional Calculus and Fractional Differential Equations, John Wiley \& Sons, New York, NY, USA, 1993.

[17] I. Podlubny, Fractional Differential Equations, Mathematics in Science and Engineering, vol. 198, Academic Press, San Diego, Calif, USA, 1999.

[18] A. A. Kilbas, H. M. Srivastava, and J. J. Trujillo, Theory and Applications of Fractional Differential Equations, vol. 204, Elsevier, Amsterdam, The Netherlands, 2006.

[19] Z. Bai and H. Lü, "Positive solutions for boundary value problem of nonlinear fractional differential equation," Journal of Mathematical Analysis and Applications, vol. 311, no. 2, pp. 495-505, 2005.

[20] J. J. Nieto and R. Rodríguez-López, “Contractive mapping theorems in partially ordered sets and applications to ordinary differential equations," Order, vol. 22, no. 3, pp. 223-239, 2005.

[21] J. Caballero, J. Harjani, and K. Sadarangani, "On existence and uniqueness of positive solutions to a class of fractional boundary value problems," Boundary Value Problems, vol. 2011, 25 pages, 2011. 


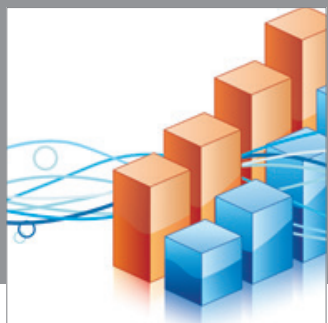

Advances in

Operations Research

mansans

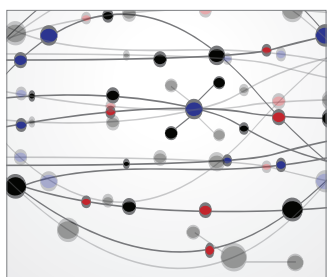

The Scientific World Journal
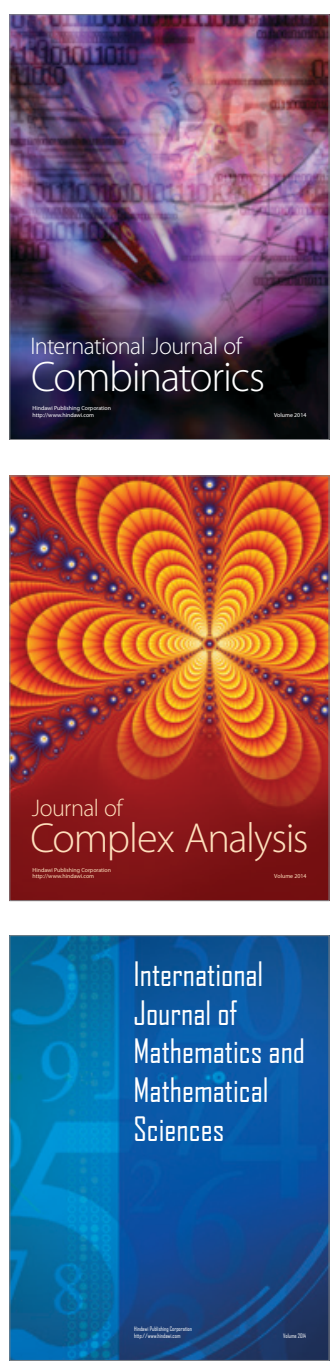
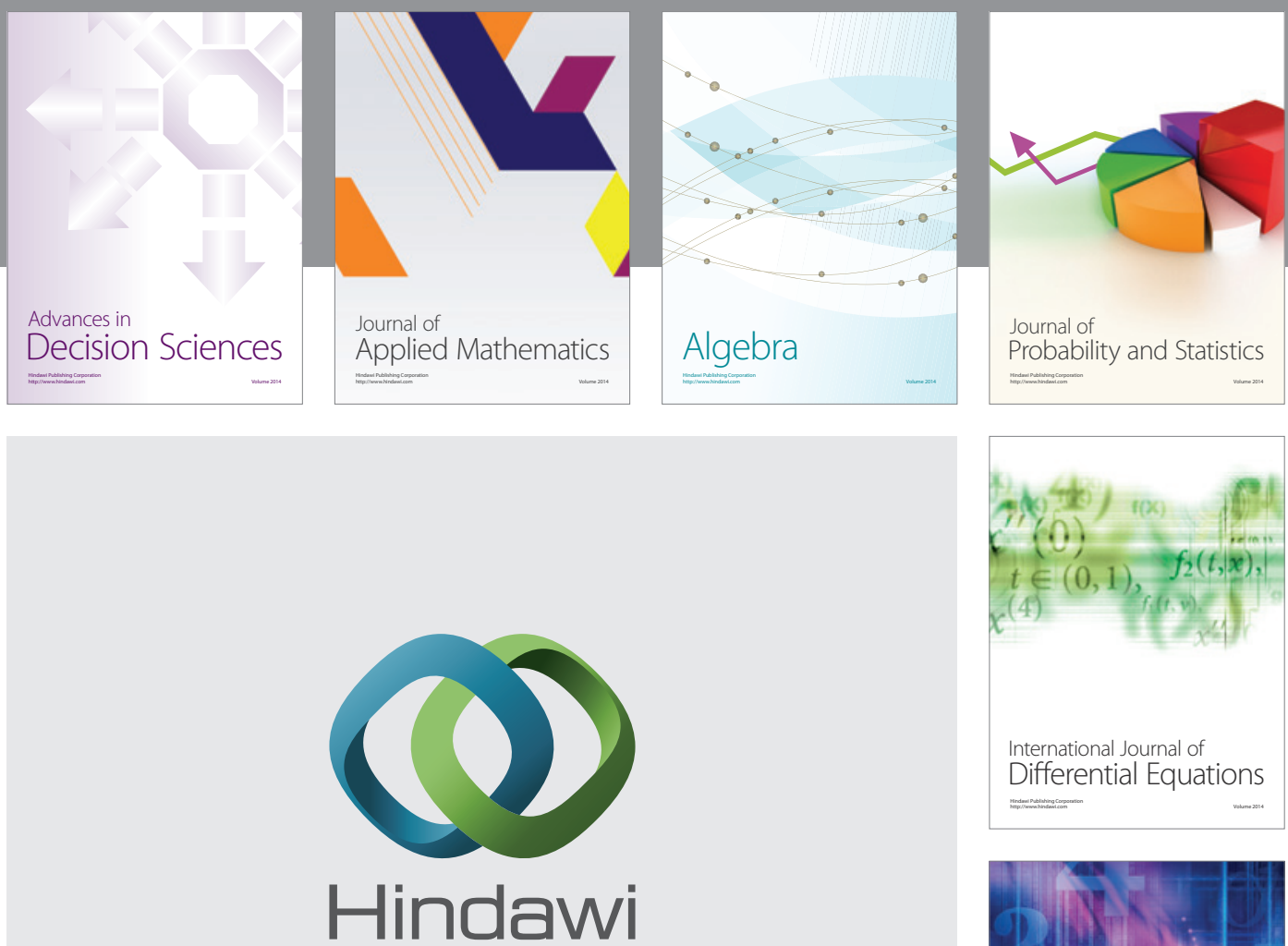

Submit your manuscripts at http://www.hindawi.com
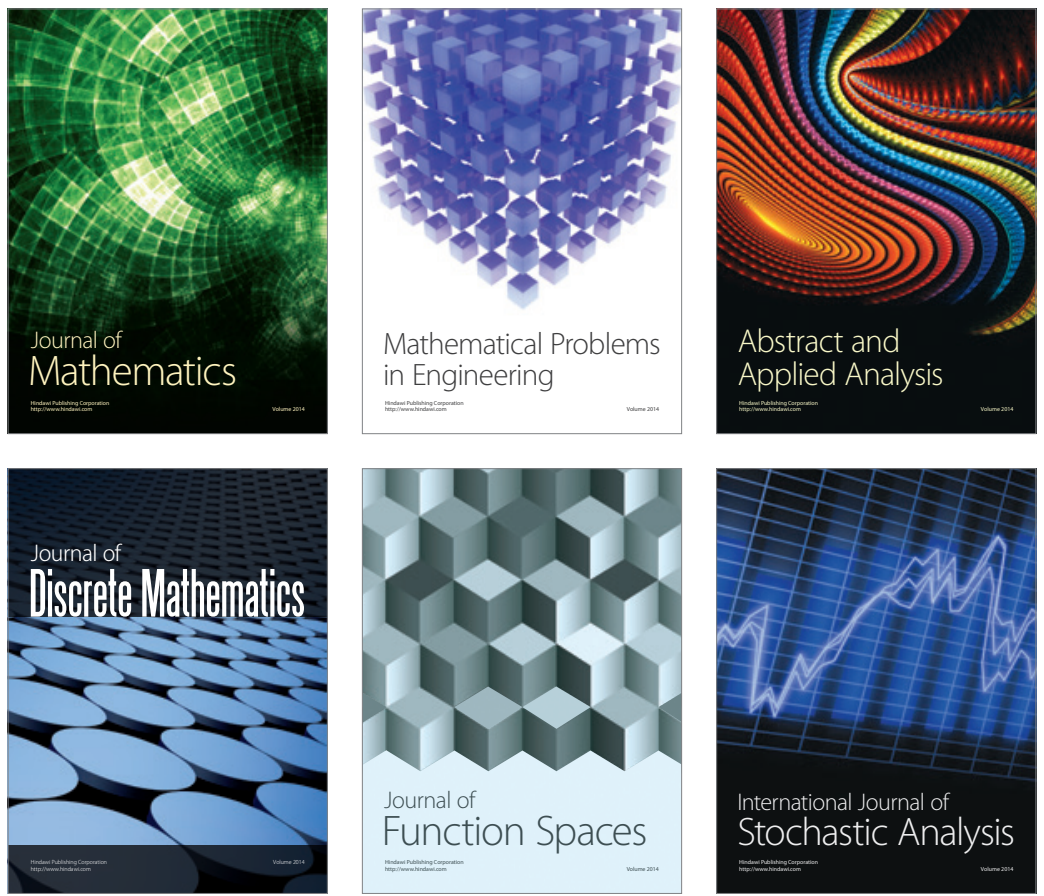

Journal of

Function Spaces

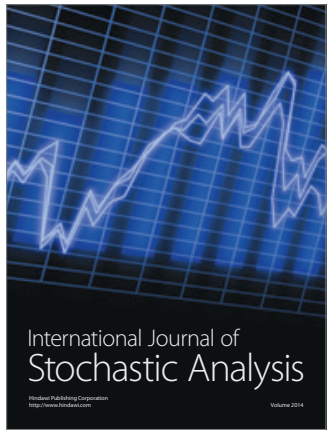

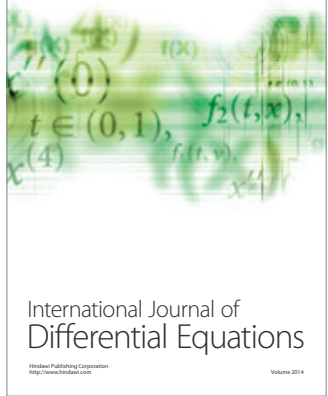
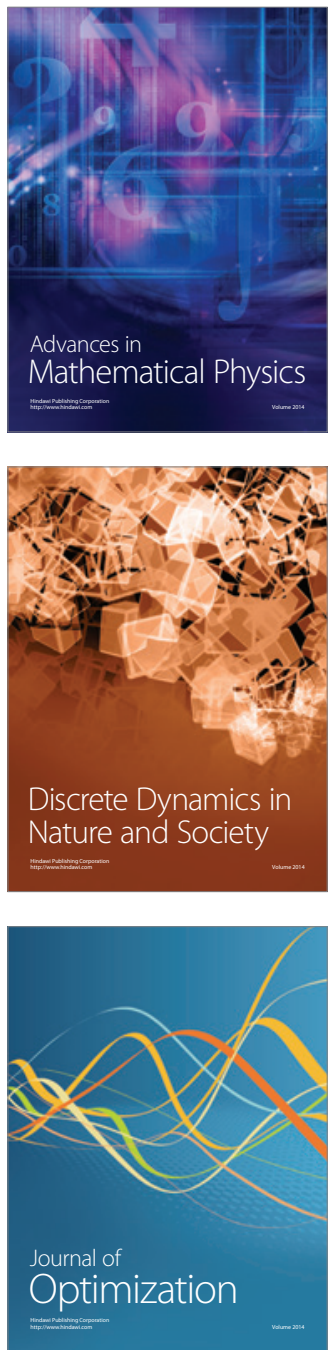